\section{Efficacy and safety of a new nutraceutical formulation in managing patients with symptomatic uncomplicated diverticular disease: a 12-month, prospective, pilot study}

\section{To the Editor,}

Diverticulosis of the colon is the most frequent endoscopic alteration detected during colonoscopy [1], and about $20 \%$ of those patients develop symptoms linked to diverticula, called Symptomatic Uncomplicated Diverticular Disease (SUDD) [2]. Although SUDD is characterized by symptoms similar to those occurring in Irritable Bowel Syndrome (IBS) (i.e. abdominal pain, bloating, alteration of bowel habits), current data showed that a combination of clinical and laboratory data may easily differentiate SUDD from IBS [3]. Pathogenesis of SUDD is not completely understood, even if dysbiosis and microscopic inflammation seem to play an important role [2]. Consequently, there is no standard treatment for SUDD, which is generally treated with non-absorbable antibiotics, anti-inflammatory drugs or probiotics [4]. In the last years, nutraceutical products have been accepted as a safer alternative/supplementation to conventional therapy. In vitro and animal model studies have shown their involvement in several biological processes, including antioxidant defenses, cell proliferation, gene expression, which may account for a role in the maintenance of the mucosal barrier integrity, the control of the inflammatory pathways and the modulation of the immune response [5]. These data suggest a wide spectrum of positive effects exerted by nutraceuticals, with a high potential for a therapeutic use in humans. Since low-grade inflammation seems to play a role in SUDD too, as well as in the occurrence of left-lower abdominal pain [6], we aimed to assess the efficacy and safety of a new nutraceutical formulation in treating patients with SUDD.

Fifteen consecutive patients ( 7 males and 8 females; mean age 63.5 years, range $57-70$; body mass index $25.5 \mathrm{~kg} / \mathrm{m}^{2}$; range 24.0-26.3) at the first diagnosis of SUDD were enrolled. All patients underwent a colonoscopy and were scored according to the Diverticular Inflammation and Complications Assessment
(DICA) endoscopic classification [7]. In particular, 14 patients were classified as DICA 1 and 1 patient as DICA 2. Patients were treated with DIVER $100^{\circ}$ (an association of natural active ingredients, including Boswellia serrata, inulin, niacin, cranberry, vitamin B1, B2, B6, B12, zinc and folic acid) 2 capsules/day for 10 days per month, and prospectively followedup for 12 months. Assessed symptoms were abdominal pain, meteorism, constipation, and diarrhea, scored by using a quantitative symptomatic scale [8]. Patients were assessed at entry, and at 3, 6, 9, and 12-month follow-up. The primary end-point was the efficacy in reducing symptoms; secondary end-points were safety and prevention of acute diverticulitis occurrence. Abdominal pain was the most relevant symptom.

Fourteen patients concluded the study, and one was lost during follow-up. During follow-up, therapy was significantly effective in reducing all symptoms with respect to baseline (Fig. 1). No adverse events were recorded during the study. No acute diverticulitis occurred except in one patient, scored as DICA 2 at the time of enrolment.

This prospective, pilot study showed that DIVER $100^{\circ}$ is effective in reducing symptoms in SUDD patients with a mild form of disease (i.e. DICA 1). In fact, all symptoms were reduced, including the most frequent and characteristic one: abdominal pain. This may be explained by the specific anti-inflammatory role of this nutraceutical formulation. For example, Boswellia serrata and cranberry have been found effective in controlling experimental colitis, thanks to their anti-oxidant and antiinflammatory properties $[9,10]$. The same anti-inflammatory effect may be exerted by other components of this nutraceutical formulation. For example, folic acid may help enhancing the activity of regulatory $\mathrm{T}$ cells [11], and vitamin $\mathrm{B} 6$ may reduce the inflammation both enhancing interleukin 10 activity [12] and increasing the growth of Lactobacilli strains, a bacterial species that seem to be effective in controlling SUDD symptoms [8]. Another strength of this nutraceutical formulation is that it seems extremely safe, since no adverse events were recorded. This result implies that this formulation may be safely used also in patients with comorbidity or intolerant to other treatments, such as mesalazine. 

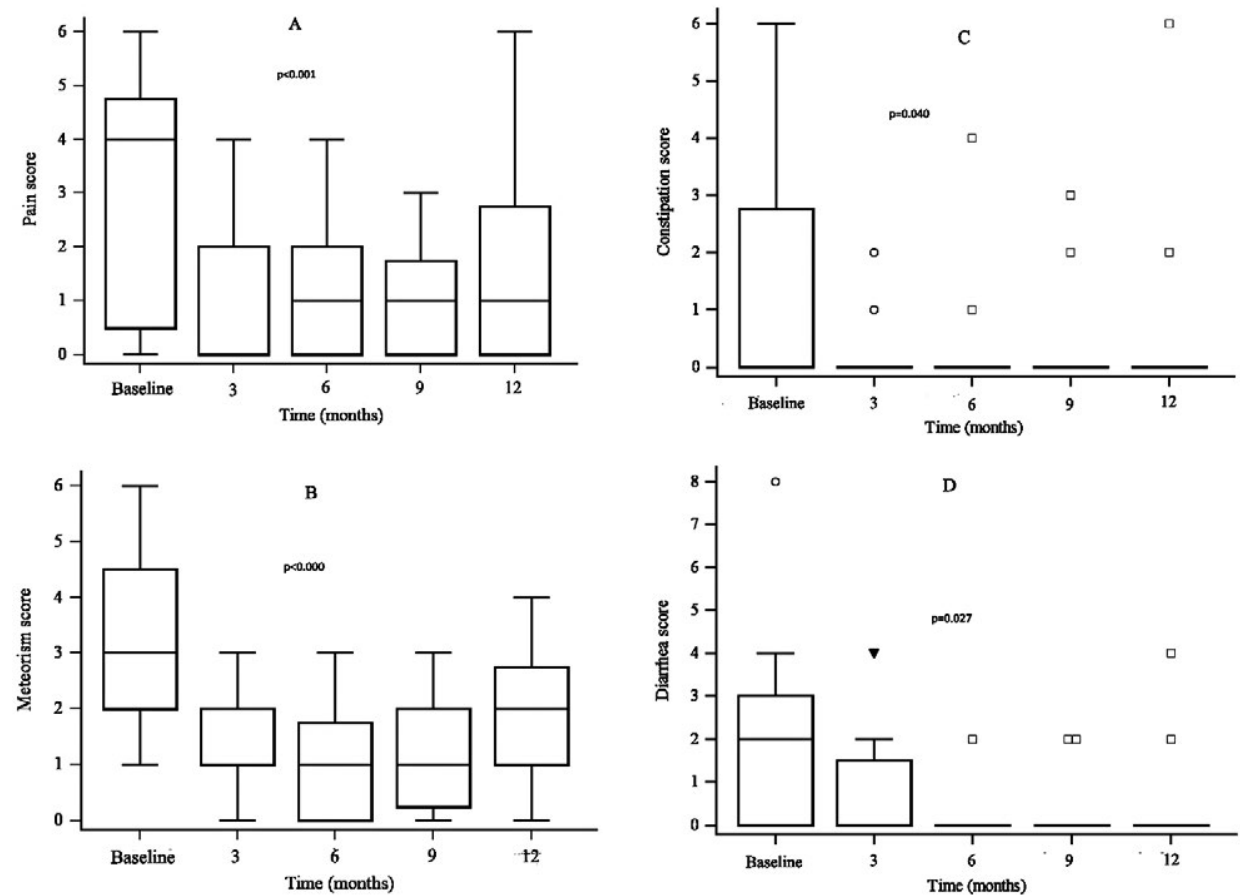

Fig. 1. Symptom scores at baseline and throughout the follow-up. A) abdominal pain; B) meteorism; C) constipation; D) diarrhea. The central box represents the interquartile range; the middle line represents the median; error bars extend from the minimum to the maximum value, excluding „outside” and „far out" values, which are displayed as separate points. Friedman test.

In conclusion, the nutraceutical formulation DIVER $100^{\circ}$ seems to be a promising therapy to control symptoms in SUDD patients. Further larger studies are warranted to confirm these preliminary data.

Antonio Tursi ${ }^{1}$, Giovanni Brandimarte ${ }^{2}$, Francesco Di Mario ${ }^{3}$, Walter Elisei ${ }^{4}$, Marcello Picchio ${ }^{5}$

1) Gastroenterology Service, ASL BAT, Andria (BT); 2) Division of Internal Medicine and Gastroenterology, "Cristo Re" Hospital, Rome; 3) Department of Clinical \& Experimental Medicine, Gastroenterology Unit, University of Parma, Parma; 4) Division of Gastroenterology, ASL RM6, Albano Laziale, Rome; 5) Division of Surgery, "P. Colombo" Hospital, ASL RM6, Velletri, Rome, Italy

Correspondence: Antonio Tursi; antotursi@tiscali.it

Conflicts of interest: None.

DOI: $10.15403 /$ jgld.2014.1121.272.fef

\section{REFERENCES}

1. Peery AF, CrockettSD, Barritt AS, etal. Burden of Gastrointestinal,Liver, and Pancreatic Diseases in the United States. Gastroenterology 2015;149:17311741.e3. doi:10.1053/j.gastro.2015.08.045

2. Tursi A, Papa A, Danese S. Review article: the pathophysiology and medical management of diverticulosis and diverticular disease of the colon. Aliment Pharmacol Ther 2015;42:664-684. doi:10.1111/apt.13322

3. Tursi A. Diverticular disease of the colon and irritable bowel syndrome: it is time to differentiate. Am J Gastroenterol 2015;110:774-775. doi:10.1038/ajg.2015.78
4. Tursi A, Picchio M, Elisei W, Di Mario F, Scarpignato C, Brandimarte G. Current Management of Patients with Diverticulosis and Diverticular Disease: A Survey from the 2nd International Symposium on Diverticular Disease. J Clin Gastroenterol 2016;50 Suppl 1:S97-S100. doi:10.1097/MCG.0000000000000645

5. Larussa T, Imeneo M, Luzza F. Potential role of nutraceutical compounds in inflammatory bowel disease. World J Gastroenterol 2017;23:24832492. doi:10.3748/wjg.v23.i14.2483

6. Tursi A, Elisei W, Picchio M, Giorgetti GM, Brandimarte G. Moderate to severe and prolonged left lower-abdominal pain is the best symptom characterizing symptomatic uncomplicated diverticular disease of the colon: a comparison with fecal calprotectin in clinical setting. J Clin Gastroenterol 2015;49:218-221. doi:10.1097/ MCG.0000000000000094

7. Tursi A, Brandimarte G, Di Mario F, et al. Development and validation of an endoscopic classification of diverticular disease of the colon: the DICA classification. Dig Dis 2015;33:68-76. doi:10.1159/000366039

8. Tursi A, Brandimarte G, Elisei W, et al. Randomised clinical trial: mesalazine and/or probiotics in maintaining remission of symptomatic uncomplicated diverticular disease--a double-blind, randomised, placebo-controlled study. Aliment Pharmacol Ther 2013;38:741-751. doi:10.1111/apt.12463

9. Hartmann RM, Fillmann HS, Martins MI, Meurer L, Marroni NP. Boswellia serrata has beneficial anti-inflammatory and antioxidant properties in a model of experimental colitis. Phytother Res 2014;28:1392-1398. doi:10.1002/ptr.5142

10. Monk JM, Lepp D, Zhang CP, et al. Diets enriched with cranberry beans alter the microbiota and mitigate colitis severity and associated inflammation. J Nutr Biochem 2016;28:129-139. doi:10.1016/j. jnutbio.2015.10.014 
11. Kinoshita M, Kayama H, Kusu T, et al. Dietary folic acid promotes survival of Foxp3+ regulatory $\mathrm{T}$ cells in the colon. J Immunol 2012;189:2869-2878. doi:10.4049/jimmunol.1200420

12. Selhub J, Byun A, Liu Z, Mason JB, Bronson RT, Crott JW. Dietary vitamin B6 intake modulates colonic inflammation in the IL10-/- model of inflammatory bowel disease. J Nutr Biochem 2013;24:2138-2143. doi:10.1016/j.jnutbio.2013.08.005

\section{Severe hyponatremia after direct-acting antiviral treatment in a patient with virus C compensated liver cirrhosis and kidney transplant}

\section{To the Editor,}

We have read with interest the case presented by Calvanese et al. [1], regarding sustained virological response (SVR) after a 4-week treatment with ritonavir-boosted paritaprevir, ombitasvir and dasabuvir (3D regimen) plus ribavirin in a kidney transplant (KT) recipient. As there is little experience focusing on this associated pathology, we present a similar case of SVR in a 58 year-old male patient with kidney transplant (KT) and hepatic $\mathrm{C}$ virus (HCV) compensated liver cirrhosis, in whom severe hyponatremia required the antiviral treatment discontinuation after 32 days. The patient was diagnosed with chronic HCV infection and chronic kidney disease (CKD) in 2000. He received a KT from a living donor in 2008 requiring immunosuppressive treatment with tacrolimus, mycophenolate mofetil and prednisone. In 2017 he was diagnosed with hepatitis $\mathrm{C}$ related liver cirrhosis, Child-Pugh score $\mathrm{A}$. and started antiviral treatment with $3 \mathrm{D}$ regimen. At the 4 -week follow-up visit, the transaminases were normal and a slight increase in serum creatinine $(1.29 \mathrm{mg} / \mathrm{dl}$ vs. $1.20 \mathrm{mg} / \mathrm{dl}$ at start) was noted, without side effects. In the 5 th week of treatment the patient presented to Emergency Department with neurological symptoms (Glasgow Coma Scale 9), severe euvolemic hyponatremia ( $\mathrm{Na} 101 \mathrm{mmol} / \mathrm{l})$ and an increase in creatinine level $(1.7 \mathrm{mg} / \mathrm{dl})$, requiring cessation of antiviral medication. The syndrome of inappropriate antidiuretic hormone secretion (SIADH) was diagnosed based on: low serum osmolality (195 $\mathrm{mOsm} / \mathrm{kg})$, increased urinary osmolality $(335 \mathrm{mOsm} / \mathrm{kg})$ and low sodium level $(104 \mathrm{mEq} / \mathrm{L})$. The tacrolimus level was increased $(20 \mathrm{ng} / \mathrm{ml})$. The laboratory analysis and imaging studies performed for the differential diagnosis of hyponatremia revealed: normal glycemia, normal thyroid and adrenal function, no diuretic use, no parenteral fluid administration, no cardiac or hepatic failure, and normal brain magnetic resonance imaging. After slow rebalancing of hyponatremia, the evolution was favorable, with reversibility of the neurological symptoms and normalization of the renal function. The follow-up 12 weeks visit revealed undetectable HCV-RNA.

The direct antiviral agents (DAAs) (most regimens including sofosvubir) in patients with $\mathrm{KT}$ showed favorable results, with SVR in $97 \%$ of patients; discontinuation and side effects are similar to non-transplanted patients [2]. The $3 \mathrm{D}$ regimen proved its efficacy and safety in end-stage CKD and hemodialysis patients, without any nephrotoxicity [3].
However, few KT patients infected with $\mathrm{HCV}$ were treated with $3 \mathrm{D}$ regimen [4].

The main concern in the treatment of KT patients for $\mathrm{HCV}$ infection with $3 \mathrm{D}$ regimen is drug interactions with immunosuppressive therapy, especially with cyclosporine and tacrolimus. Ritonavir is a strong inhibitor of CYP3A4, so co-administration of other drugs metabolized by this enzyme (such as tacrolimus) may increase plasma concentrations. The current recommendation for KT patients receiving $3 \mathrm{D}$ regimen is to reduce the dose of tacrolimus with close monitoring of blood levels and dosage adjustment [4]. Only a few isolated cases in the literature described tacrolimus induced hyponatremia due to SIADH or to salt losing nephropathy, irrespective of the tacrolimus level $[5,6]$.

To the best of our knowledge, this is the first case to report hyponatremia as a severe side effect of $3 \mathrm{D}$ regimen, most likely secondary to ritonavir - tacrolimus interaction. Although the optimal duration of 3D treatment is considered 12 weeks, both cases suggested that SVR could be obtained in KT patients with a shorter duration of treatment.

Cătălina Mihai ${ }^{1}$, Bogdan Mircea Mihai $^{2}$, Mihaela Dranga ${ }^{1}$, Vasile Liviu Drug ${ }^{1}$, Adrian Covic ${ }^{3}$, Cristina Cijevschi Prelipcean ${ }^{1}$

1) Institute of Gastroenterology and Hepatology, "Grigore T. Popa" University of Medicine and Pharmacy, "Sf. Spiridon" Clinical Hospital, Iași; 2) Clinical Centre of Diabetes, Nutrition and Metabolic Diseases, "Grigore T. Popa" University of Medicine and Pharmacy, "Sf. Spiridon" Clinical Hospital, Iași; 3) Nephrology Department, Dialysis and Renal Transplant Center, "Grigore T. Popa" University of Medicine and Pharmacy, "Dr. C.I. Parhon" University Hospital Iasi, Romania

Correspondence: Bogdan Mircea Mihai; bogdanmihai2003@yahoo.com

Conflicts of interest: None.

DOI: $10.15403 /$ jgld.2014.1121.272.hyp

\section{REFERENCES}

1. Calvanese C, Lisotti A, Fiorenza S, Mandreoli M, Caletti G, Fusaroli P. Sustained virological response after 4-week ritonavir-boosted paritaprevir, ombitasvir and dasabuvir plus ribavirin treatment in a kidney transplant recipient. J Gastrointestin Liver Dis 2017;26:320-321. doi:10.15403/jgld.2014.1121.263.frz

2. Chute DF, Chung RT, Sise ME. Direct-acting antiviral therapy for hepatitis $\mathrm{C}$ virus infection in the kidney transplant recipient. Kidney Int 2018;93:560-567. doi:10.1016/j.kint.2017.10.024

3. Pockros PJ, Reddy KR, Mantry PS, et al. Efficacy of Direct-Acting Antiviral Combination for Patients With Hepatitis C Virus Genotype 1 Infection and Severe Renal Impairment or End-Stage Renal Disease. Gastroenterology 2016;150:1590-1598. doi:10.1053/j. gastro.2016.02.078

4. Cholongitas E, Pipili C, Papatheodoridis GV. Interferon-free regimens in patients with hepatitis $\mathrm{C}$ infection and renal dysfunction or kidney transplantation. World J Hepatol 2017;9:180-190. doi:10.4254/wjh. v9.i4.180

5. Bagchi S, Husain Zaidi S, Prasad Mathur R. Severe symptomatic hyponatremia - an uncommon presentation of tacrolimus nephrotoxicity. Nephrol Dial Transplant 2011;26:2042-2044. doi:10.1093/ndt/gfr133 
6. Takagi K, Yagi T, Shinoura S, et al. Syndrome of Inappropriate Antidiuretic Hormone Secretion Following Liver Transplantation. Acta Med Okayama 2017;71:85-89. doi:10.18926/AMO/54830

\section{Liver stiffness better predicts portal hypertension after HCV eradication}

\section{To the Editor,}

Eradication of $\mathrm{HCV}$ infection with direct acting antiviral (DAA) therapy in patients with compensated advanced chronic liver disease (cACLD) brings new questions regarding the reliability and the accuracy of noninvasive tests in the postHCV settings. Recent data reported that liver stiffness rapidly decreases especially during the first 4 weeks of DAA therapy, with no notable changes at the end of treatment (EOT) or when assessing the sustained virologic response (SVR) at 12 weeks after EOT [1]. Data from the Peg-Interferon era showed that baseline HVPG (hepatic-vein portal gradient), irrespective of achieving SVR, is the sole predictor of longterm decompensation and mortality [2]. As liver stiffness measurement (LSM) is considered a very good predictor of clinically significant portal hypertension (CSPH) [3, 4], we investigated the accuracy of LSM to estimate CSPH and to predict long-term outcome in CACLD patients after obtaining SVR.

Sixty-nine consecutive HCV patients with cACLD (mean age $59.2 \pm 8.2$ years; $63.8 \%$ females) eligible for DAA therapy were included. They all had HVPG measurement at baseline and at SVR12. Transient elastography was used to measure the LSM at baseline, EOT and SVR12. All patients obtained viral clearance. During the study period, median LSM decreased from $23.15(6.4-62.7) \mathrm{kPa}$ at baseline to $18.7(7.3-47.2) \mathrm{kPa}$ at EOT $(\mathrm{p}=0.015)$ and remained unchanged at SVR12 [19.8 (5.9-55.1) $\mathrm{kPa} ; \mathrm{p}=0.37]$. Median HVPG dropped from 12.5 (327) $\mathrm{mmHg}$ at baseline to 9 (3-27) $\mathrm{mmHg}$ at SVR12, $\mathrm{p}=0.013$. The proportion of patients with CSPH decreased from $74.2 \%$ at baseline to $46.7 \%$ at SVR12 (chi-square=13.48; $<<0.001$ ). Interestingly, the correlation between LSM and HVPG was stronger at SVR12 $(r=0.839 ; \mathrm{p}<0.001)$ as compared to baseline $(r=0.698 ; p<0.001)$. Furthermore, the performance of LSM to detect $\mathrm{CSPH}$ was better after obtaining viral clearance [AUROC of 0.88 (95\%CI: 0.82-1.00) vs. 0.82 (95\%CI: 0.70 0.94 ) and diagnostic accuracy of $80 \%$ vs $57.6 \%$ ] and the best cut-off values (calculated using the Youden index) were different: $12 \mathrm{kPa}$ at SVR12 vs. $20.5 \mathrm{kPa}$ at baseline.

Despite the small number of patients included in this single center and still on-going experience, the results are worthy of careful analysis. First, we demonstrated that the DAA therapy induced a reduction of LSM during treatment that was maintained after 12 weeks of discontinuation, proving the significant effect of HCV-induced liver inflammation on LSM [1]. The reduction of HVPG also proved the important role of $\mathrm{HCV}$-induced inflammation on hepatic vascular resistance. The particular behavior of LSM and HVPG after DAA therapy is in accordance with the recent results published by Lens et al. [5], showing a better diagnostic performance of LSM for CSPH after DAA therapy [5]. The different LSM cut-off values obtained for the prediction of CSPH seem confusing only at first sight; at a closer look, they are in line with the findings that proposed different thresholds for ruling-in and out CSPH [5]. Indeed, taking into account that the baseline LSM cut-off value of $20.5 \mathrm{kPa}$ had a $91 \%$ positive predictive value, it could be used to rule-in CSPH; the SVR12 LSM cut-off value of 12 $\mathrm{kPa}$ has a $100 \%$ negative predictive value, being more suitable to rule-out CSPH, especially in the absence of HCV infection.

The HCV clearance due to DAA therapy induces a reduction in LSM and HVPG, mainly due to the elimination of the inflammatory component of hepatic stiffness and vascular resistance. Our results show that, in the absence of HCV infection at SVR, LSM and HVPG seem to correlate better and LSM seems to be a better noninvasive tool to rule-out than to rule in CSPH in infected patients.

Corina Radu ${ }^{1,2^{*}}$, Oana Stancu ${ }^{3,4^{*}}$, Roxana $\mathrm{Sav}^{3}$, Anca Bugariu ${ }^{2,3}$, Alina Suciu ${ }^{1,2}$, Crina Grigoras ${ }^{3}$, Bogdan Procopet ${ }^{1,2,3}$, Horia Stefanescu $^{2,3}$, Zeno Sparchez ${ }^{1,2}$

1) 3rd Medical Clinic, Department of Internal Medicine, Iuliu Hatieganu University of Medicine and Pharmacy; 2) Hepatology Unit, Regional Institute of Gastroenterology and Hepatology; 3) Liver Research Club, Iuliu Hatieganu University of Medicine and Pharmacy, Cluj-Napoca; 4) Central Military Hospital, Carol Davila University of Medicine and Pharmacy, Bucharest, Romania * equal contribution

Correspondence: Horia Stefanescu; horia.stefanescu@irgh.ro

Financial support: This work was financed in part by an internal grant from the Iuliu Hatieganu University of Medicine and Pharmacy (4944/3/08.03.2016) awarded to Corina Radu.

Conflicts of interest: None.

DOI: $10.15403 /$ jgld.2014.1121.272.hcv

\section{REFERENCES}

1. Pons M, Santos B, Simón-Talero M, et al. Rapid liver and spleen stiffness improvement in compensated advanced chronic liver disease patients treated with oral antivirals. Therap Adv Gastroenterol 2017;10:619-629. doi:10.1177/1756283X17715198

2. Lens S, Rincón D, García-Retortillo M, et al. Association Between Severe Portal Hypertension and Risk of Liver Decompensation in Patients With Hepatitis C, Regardless of Response to Antiviral Therapy. Clin Gastroenterol Hepatol 2015;13:1846-1853.e1. doi:10.1016/j. cgh.2015.04.013

3. European Association for Study of Liver; Asociacion Latinoamericana para el Estudio del Higado. EASL-ALEH Clinical Practice Guidelines: Non-invasive tests for evaluation of liver disease severity and prognosis. J Hepatol 2015;63:237-264. doi:10.1016/j.jhep.2015.04.006

4. de Franchis R; Baveno VI Faculty. Expanding consensus in portal hypertension Report of the Baveno VI Consensus Workshop: Stratifying risk and individualizing care for portal hypertension. J Hepatol 2015;63:743-752. doi:10.1016/j.jhep.2015.05.022

5. Lens S, Alvarado-Tapias E, Mariño Z, et al. Effects of All-Oral AntiViral Therapy on HVPG and Systemic Hemodynamics in Patients With Hepatitis C Virus-Associated Cirrhosis. Gastroenterology 2017;153(5):1273-1283.e1. doi:10.1053/j.gastro.2017.07.016 


\section{Obesity and irritable bowel syndrome: coincidence or association?}

\section{To the Editor,}

Irritable bowel syndrome (IBS) is a medical condition able to alter the eating patterns and thus susceptible to induce body mass index (BMI) changes. It has been reported that IBS is more common in patients with morbid obesity [1]. Although the reason for this association is unknown, some hypothesis may be formulated. Altered small bowel and colonic transit and low-fiber and high-refined-carbohydrate diets are linked to obesity and are potential contributors to this association. More than $60 \%$ of the people with IBS complain of food intolerance, unable to change the diet and predisposing to both obesity and IBS symptoms [2]. Altered absorption or metabolism of fat may also contribute [3]. A comprehensive review was not able to detect any significant association between obesity and IBS because of the heterogeneity of the studies [4], confirming the high heterogeneity of IBS populational studies [5].

Given this status of information, we looked into our database and analyzed phenotypic characteristics and the relationship between obesity (expressed by BMI) and IBS, stratified in two groups: one with non-post-infectious IBS (NPI-IBS) and the other one with post-infectious IBS (PI-IBS). We identified 592 IBS patients: 528 patients with NPI-IBS (174 males, 354 females), of whom 99 were obese ( 34 males, 65 female, mean \pm SD age $45.8 \pm 5$ years) and 64 PI-IBS patients (25 males, 39 females) of whom 17 were obese ( 6 males, 11 females, mean \pm SD age $41.7 \pm 5$ years). We compared these groups with a control group of 100 age and sex matched patients, randomly selected from the hospital database after exclusion of functional gastrointestinal pathology ( 39 male, 61 female), of whom 22 were obese patients ( 9 male, 13 female) (Table I). The prevalence of obesity in NPI-IBS was $18.75 \%$ vs. $22 \%$ in the control group $\left(\chi^{2}: 0.5709, p=0.75\right)$; in PI-IBS obesity was present in $26.57 \%\left(\chi^{2}: 0.4482, \mathrm{p}=0.79\right)$.

Thus, in our large IBS sample, obesity is as frequently encountered in IBS as in the general population.

According to our data, underweight patients from the control group are almost twice the number of NPI-IBS and PIIBS patients taken together. There is no significant difference between the three groups of patients with normal weight. In each IBS group, the female gender with class I obesity was predominant: 59/99 NPI-IBS (59.6\%) and 9/17 PI-IBS (52.94\%), respectively. Bloating and flatulence were more common in both obese IBS groups, $57 \%$ in NPI-IBS and $41 \%$ in PI-IBS compared to $23 \%$ in the control group. In the NPI-IBS group, the therapeutic response at one month (probiotics, prebiotics, intestinal anti-inflammatory drugs, antibiotics, opioids, 5-HT 3 receptor antagonists, antispasmodics in monotherapy or in combination) failed in $37 \%$ of NPI-IBS obese patients as
Table I. Distribution of the patients based on their body mass index (BMI)

\begin{tabular}{lccc}
\hline Body Mass Index $\left(\mathrm{kg} / \mathrm{m}^{2}\right)$ & $\begin{array}{c}\text { NPI-IBS } \\
(\mathrm{n}=528)\end{array}$ & $\begin{array}{c}\text { PI-IBS } \\
(\mathrm{n}=64)\end{array}$ & $\begin{array}{c}\text { Controls } \\
(\mathrm{n}=100)\end{array}$ \\
\hline Underweight n (\%) & $12(2.46 \%)$ & $2(3.12 \%)$ & $6(6 \%)$ \\
Normal18.50 -24.99 $\mathrm{n}(\%)$ & $221(41.85 \%)$ & $29(45.31 \%)$ & $52(52 \%)$ \\
Overweight25 -29.99n (\%) & $196(37.12 \%)$ & $16(25 \%)$ & $20(20 \%)$ \\
$\begin{array}{l}\text { Obesity class I 30-34.9 } \\
\mathrm{n}(\%)\end{array}$ & $57(10.78 \%)$ & $6(9.37 \%)$ & $12(12 \%)$ \\
$\begin{array}{l}\text { Obesity class II 30-34.9 } \\
\mathrm{n}(\%)\end{array}$ & $31(5.87 \%)$ & $10(15.62 \%)$ & $8(8 \%)$ \\
Obesity class III $\geq 40 \mathrm{n}(\%)$ & $11(2,08 \%)$ & $1(1.58 \%)$ & $2(2 \%)$ \\
\hline
\end{tabular}

against $23 \%$ of NPI-IBS normal weight patients assessed by the physician, ( $\chi 2: 6.8987, \mathrm{p}: 0.0317)$ and in $66 \%$ PI-IBS obese versus $54 \%$ non obese PI-IBS patients ( $\chi 2$ :0.6968, p:0.705803).

In conclusion, in our study obesity had a similar prevalence in NPI-IBS and PI-IBS as in controls. However, patients with PI-IBS and class II obesity were more numerous than NPIIBS and controls having similar BMI (15.62\% vs. $8 \%$ in the control group and $5.87 \%$ in NPI-IBS group). Most NPI-IBS obese patients $(10.78 \%)$ and obese controls (12\%) belonged to class I obesity. The therapeutic response was less favorable in NPI-IBS and PI-IBS obese patients than in NPI-IBS and PI-IBS normal weight patients.

\section{Flaviu Rusu}

2nd Department of Internal Medicine, Iuliu Hatieganu University of Medicine and Pharmacy, Cluj-Napoca, Romania

Correspondence: Flaviu Rusu, flaviurusu@yahoo.com

Conflicts of interest: None.

DOI: $10.15403 /$ jgld.2014.1121.272.ibs

\section{REFERENCES}

1. Schneck AS, Anty R, Tran A, et al. Increased Prevalence of Irritable Bowel Syndrome in a Cohort of French Morbidly Obese Patients Candidate for Bariatric Surgery. Obes Surg 2016;26):1525-1530. doi:10.1007/s11695-015-1907-0

2. Eswaran S, Tack J, Chey WD. Food: the forgotten factor in the irritable bowel syndrome. Gastroenterol Clin North Am 2011;40:141-162. doi:10.1016/j.gtc.2010.12.012

3. Aasbrenn M, Høgestøl I, Eribe I, et al. G. Prevalence and predictors of irritable bowel syndrome in patients with morbid obesity: a crosssectional study. BMC Obes 2017;4:22. doi:10.1186/s40608-017-0159-Z

4. Pickett-Blakely O. Obesity and irritable bowel syndrome: a comprehensive review. Gastroenterol Hepatol (N Y) 2014;10:411-416.

5. Plavšić I, Hauser G, Tkalčić M, Pletikosić S, Salkić N. Diagnosis of Irritable Bowel Syndrome: Role of Potential Biomarkers. Gastroenterol Res Pract 2015;2015:490183. doi:10.1155/2015/490183 